\title{
Clinical Efficacy of Endocytoscopy for Gastrointestinal Endoscopy
}

\author{
Masashi Misawa', Shin-ei Kudo', Yuki Takashina', Yoshika Akimoto ${ }^{1}$, Yasuharu Maeda', Yuichi Mori ${ }^{1}{ }^{1,2}$, Toyoki Kudo ${ }^{1}$, Kunihiko \\ Wakamura', Hideyuki Miyachi', Fumio Ishida' and Haruhiro Inoue ${ }^{3}$ \\ ${ }^{1}$ Digestive Disease Center, Showa University Northern Yokohama Hospital, Yokohama, Japan, ${ }^{2}$ Clinical Effectiveness Research Group, \\ Institute of Health and Society, University of Oslo, Oslo, Norway, ${ }^{3}$ Digestive Disease Center, Showa University Koto Toyosu Hospital, \\ Tokyo, Japan
}

Endocytoscopy (EC) is a contact-type optical endoscope that allows in vivo cellular observation during gastrointestinal endoscopy and is now commercially available not only in Japan but also in Asian, European Union, and Middle Eastern countries. EC helps conduct a highly accurate pathological prediction without biopsy. Initially, EC was reported to be effective for esophageal diseases. Subsequently, its efficacy for stomach and colorectal diseases has been reported. In this narrative review, we searched for clinical studies that investigated the efficacy of EC. EC seems to accurately diagnose gastrointestinal diseases without biopsy. Most of the studies aimed to clarify the relationship between endocytoscopic findings of gastrointestinal neoplasia and pathological diagnosis. Some studies have investigated non-epithelial lesions or diseases, such as inflammatory bowel disease or infectious diseases. However, there are few high-level pieces of evidence, such as randomized trials; thus, further studies are needed. Clin Endosc 2021;54:455-463

Key Words: Endocytoscopy; Lower gastrointestinal endoscopy; Magnifying endoscopy; Upper gastrointestinal endoscopy

\section{INTRODUCTION}

What is the goal of endoscopic diagnosis? One of these goals is to achieve accurate diagnoses comparable to pathological diagnoses without biopsy. To address this issue, an ultra-magnification endoscopy system was developed to visualize cellular level images in vivo. Ultra-magnifying endoscopy aims to conduct an 'optical biopsy' that provides an accurate pathological prediction based on a histologic-equivalent image. ${ }^{1}$ There are two methods to vitalize the cellular image; the first is confocal laser endomicroscopy and the second is endocytoscopy (EC).

Received: May 21, 2021 Revised: June 7, 2021

Accepted: June 10, 2021

Correspondence: Masashi Misawa

Digestive Disease Center, Showa University, Showa University Northern Yokohama Hospital, 35-1 Chigasaki-chuo, Tsuzuki, Yokohama 224-8503, Japan

Tel: +81-45-949-7573, Fax: +81-45-949-7263, E-mail: mmisawa@med.showa-u. ac.jp

ORCID: https://orcid.org/0000-0002-8520-2036

It is the invited review article.

(c) This is an Open Access article distributed under the terms of the Creative Commons Attribution Non-Commercial License (http://creativecommons.org/ licenses/by-nc/3.0) which permits unrestricted non-commercial use, distribution, and reproduction in any medium, provided the original work is properly cited.
In 1982, Tada et al., in collaboration with Olympus, developed a magnifying endoscope (contact-type fiberscope) with a switchable lens (rotating turret) that increased the magnification of the optical lens system by approximately 170 times and attempted to observe the colonic mucosa. To the best of our knowledge, this is the first report of in vivo cellular observation in gastrointestinal endoscopy. In 2004, the first reports of a video-endoscopy-based EC system were reported by Inoue and Kumagai. ${ }^{1,2}$ The prototype EC system was developed by Olympus Corporation and was a type of catheter with a diameter of $3.4 \mathrm{~mm}$. Both studies focused on esophageal squamous cells and successfully visualized the cellular atypia of esophageal squamous cell carcinoma. The first prospective study using EC was for colorectal lesions. ${ }^{3}$ Sasajima et al. ${ }^{3}$ reported 113 consecutive lesions observed by catheter-type EC. Their study had two important findings: the first was that EC could visualize the cellular atypia of the colorectal lesion as well as the esophagus, and the second was that the diagnosis based on visualized cellular atypia correlated with the pathological diagnosis. These studies brought about the development of endocytoscopic research. In this review, we focused on the development and efficacy of EC for gastrointestinal diseases. Although this was a narrative review, we searched the eligible 
articles from PubMed based on the following search terms: (endocytoscopy) and (stomach or esophagus or duodenum or colon or colorectum or colonoscopy). Review articles and case reports were excluded.

\section{SPECIFICATION OF ENDOCYTOSCOPES AND STANDARD OBSERVATION TECHNIQUES}

In this section, the specifications of the commercially available endocytoscopes and standard techniques of endocytoscopic observations are described. Some prototype endocytoscopes have been reported; however, commercially available endocytoscopes for the upper and lower gastrointestinal tract (GIF-H290EC and CF-H290ECI; Olympus Corp. Tokyo, Japan) were launched in 2018., ${ }^{4,5}$ These two scopes have one objective lens with 520-fold magnification and a manual zoom mechanism similar to a conventional magnifying endoscope. One endoscope can switch from normal observation to magnified observation and ultra-magnifying observation. The detailed specifications of each scope are listed in Table 1.

Since the endocytoscope is a contact-type endomicroscope, it requires a different maneuver compared with conventional magnification. Conventional magnification endoscopy needs to maintain the distance between the lens and the target lesion to obtain a focused image. In contrast, EC does not require distance to be maintained. An ultra-magnified image can be obtained by just contacting the lesion and then pulling down the magnification lever (Supplementary Video 1). Dye staining is mandatory for visualizing cell nuclei. Minami et al. and Ichimasa et al. reported that double staining with crystal violet and methylene blue is appropriate for both upper and lower gastrointestinal EC. ${ }^{6,7}$ Supplementary Video 2 shows how to perform methylene blue staining. To maintain a clear endocytoscopic image, meticulous water cleansing is required.

\section{EC FOR ESOPHAGEAL DISEASES}

The endocytoscopic diagnosis for esophageal neoplasms has been actively investigated because, as described before, the first research on EC was performed for the esophagus. The main target of this field is esophageal squamous cell carcinoma, because early on in this academic field, almost all studies were reported in Japan, where the incidence of Barret's esophagus is relatively small compared with Western countries. Endocytoscopic diagnosis for esophageal squamous cell carcinoma seems to be accurate; however, high-level evidence is lacking in this field.

Inoue et al. reported an endocytoscopic atypia (ECA) classification, which was based on the irregularity of cell nuclei visualized by EC. ECA was classified into five categories (ECA1ECA5). ${ }^{8}$ Endocytoscopic diagnoses in accordance with the ECA classification correlated with histopathological diagnoses based on the biopsy or endoscopically resected specimens. The overall accuracy of EC for differentiating between non-malignant tissue and squamous cell carcinoma was $82 \%$. Kumagai et al. reported a similar diagnostic classification that had three categories (Type 1-Type 3) according to the iodine staining findings and EC findings. ${ }^{9}$ Interestingly, they conducted an image-reading test for endocytoscopic images by gastroenterological pathologists. In this analysis, out of 46 lesions of squamous cell carcinoma, 43 lesions did not require a pathological diagnosis. They concluded that $\mathrm{EC}$ has the potential to lower the numbers of biopsies. ${ }^{9}$ Kumagai et al. also reported that their classification provided $94.9-100 \%$ sensitivity for squamous cell carcinoma, whereas the specificity was $46.7-80.0 \%$. The lower specificity was attributed to the low magnification of the prototype endocytoscope (380-times). ${ }^{10}$ Subsequently, Kumagai reported that high magnification power (600-times) improved the diagnostic performance. ${ }^{11}$ Minami et al. reported crystal violet and methylene blue double staining, which provides an image similar to that of hematoxylin and eosin

Table 1. Specifications of the Endocytoscopes

\begin{tabular}{lcc}
\hline & CF-H290ECI & GF-H290EC \\
\hline Field of view $\left(^{\circ}\right)$ & 140 & 140 \\
Magnification power & $x 520$ & x520 \\
Distal end outer diameter $(\mathrm{mm})$ & 12.8 & 9.7 \\
Channel outer diameter $(\mathrm{mm})$ & 3.2 & 2.2 \\
Water jet & Yes & Yes \\
\hline
\end{tabular}


staining. Based on this double staining method, they conducted a comparison between endocytoscopic images and pathological diagnoses of corresponding resected specimens using Inoue's ECA classification. The sensitivity, specificity, and accuracy for squamous cell carcinoma were $91.7 \%, 91.0 \%$, and $91.3 \%$, respectively. ${ }^{7}$ Recently, Shimamura et al. reported a three-tiered modified Inoue's ECA classification (Fig. 1). They conducted an image-review test using 55 lesions. Expert and non-expert endoscopists independently reviewed only the endocytoscopic images, and their diagnoses were compared with the pathological diagnoses. The sensitivity and specificity of EC for non-neoplasia vs. neoplasia were $88.7 \%$ and $74.6 \%$, respectively, by experts and $90.3 \%$ and $52.1 \%$, respectively, by non-experts. ${ }^{12}$

There are a couple of studies regarding Barret's esophagus. ${ }^{13,14}$ Tomizawa proposed a classification system for Barret's esophagus that referred to Inoue's ECA classification. Although it was a pilot ex vivo study, their classification system provided 95.8-98.3\% accuracy for esophageal adenocarcinoma. $^{14}$

\section{EC FOR STOMACH NEOPLASMS}

Compared with the esophagus, the number of studies on $\mathrm{EC}$ of the stomach is limited. The stomach is a mucous-rich organ, and therefore, it is challenging to obtain a clear endocytoscopic image. However, some studies indicate that EC can differentiate cancerous tissue from non-neoplastic lesions with high accuracy and can also predict Helicobacter pylori infection.

The first in vivo study was reported by Eberl et al. They observed gastric neoplasia using EC with methylene blue staining. Twenty-three gastric lesions were observed, and the video sequences recorded were reviewed by two pathologists. They reported that EC could identify gastric neoplasms with a sensitivity of $56 \%$ and a specificity of $89 \% .{ }^{15}$ Isomoto et al. reported 17 cases of gastric malignant lymphoma using EC. EC can visualize the inter-glandular infiltration of lymphoma cells as smaller-sized and intensely stained nuclei. ${ }^{16}$ Chiu et al. reported that EC could identify goblet cells of the gastric mu-

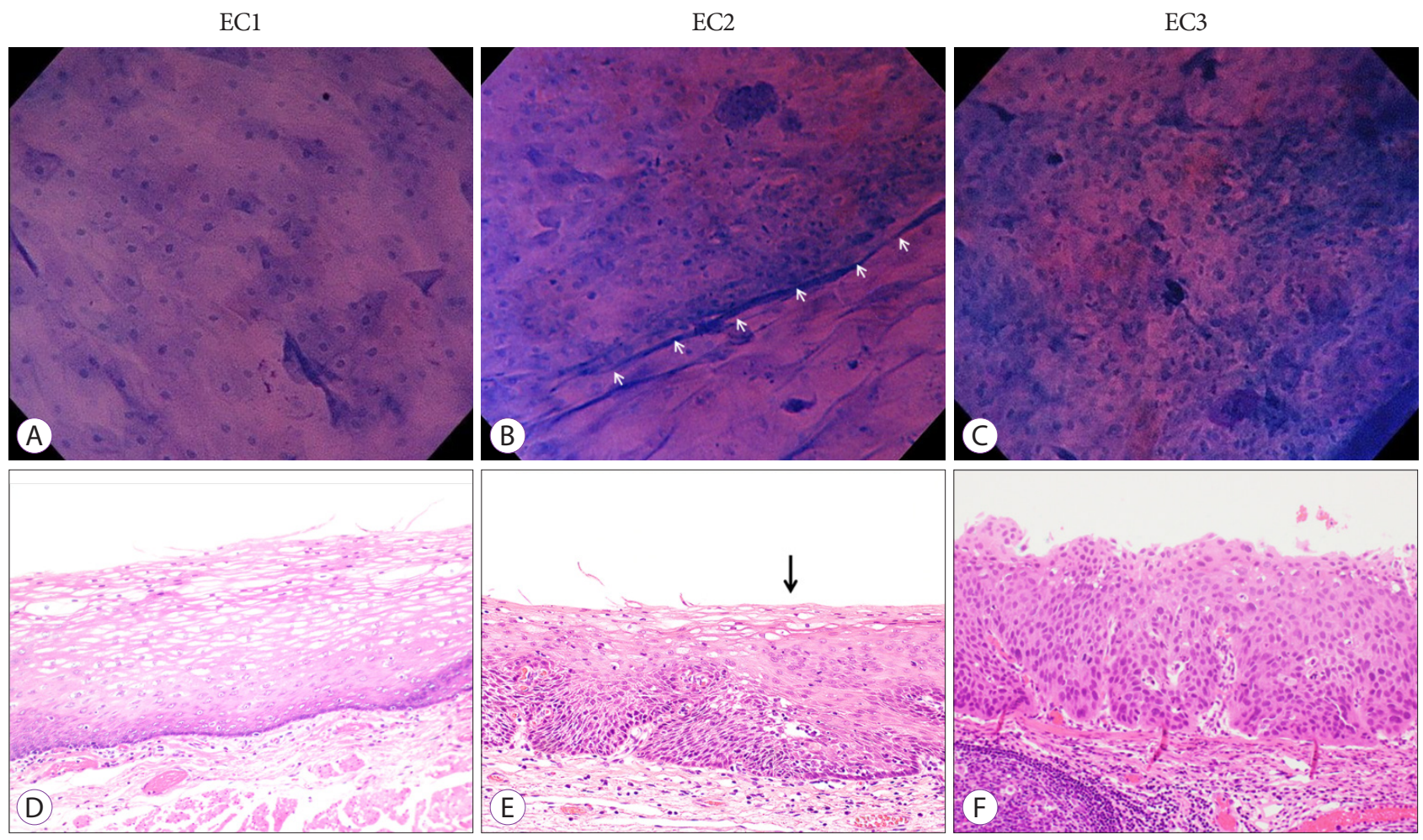

Fig. 1. Endocytoscopic classification for esophageal squamous cell carcinoma, proposed by Shimamura et al. ${ }^{11}$ Their classification has three categories. The upper row shows typical endocytoscopic images and the lower row shows corresponding hematoxylin and eosin staining. (A, D) reveals cytoplasm-rich cells with large rhomboid-shaped cells and a uniform pattern of small and round nuclei. (B, E) shows slightly higher cellular density compared with $A, D$ and the nuclei appear small or mildly enlarged. (C, F) shows a significant increase in the cellular density with loss of cellular structure along with a demarcation line, and the nuclei are enlarged with heterogeneity in size and shape. EC, endocytoscopy. 
cosa for recognition of gastric intestinal metaplasia. Although the image quality of the used prototype endocytoscope was insufficient, EC could identify goblet cells with an accuracy of $86 \%{ }^{17}$ A large-scale evaluation of the diagnostic performance of EC for gastric cancer was reported by Kaise et al. Two studies were conducted to elucidate the diagnostic performance of EC in gastric cancer. They reported that cellular atypia visualized by EC correlated well with pathological diagnosis. The overall accuracy for diagnosing gastric cancer was a sensitivity of $78.4-86 \%$ and a specificity of $93.3-100 \%{ }^{18,19}$ Sato et al. also reported the efficacy of EC. They investigated the relationship between Helicobacter pylori infection and endocytoscopic findings. Similar to Chiu's study, they focused on goblet cells and the shape of the glandular orifice. They concluded that EC can differentiate gastric mucosal patterns of minimal, non-neoplastic changes and appears to reliably exclude Helicobacter pylori infection. ${ }^{20}$

\section{EC FOR THE DUODENUM}

Reports regarding the efficacy of EC for duodenal lesions or diseases are currently limited. However, recent advancements in endoscopic treatment procedures, including full-thickness resection for duodenal neoplasms, have further increased the importance of pre-treatment diagnosis. Therefore, a precise optical biopsy of duodenal lesions is needed; therefore, EC for duodenal diseases should be investigated.

The first study on the efficacy of EC for duodenal diseases was on celiac sprue. Pohl et al. investigated whether endocytoscopic images correlated with the disease activity of celiac sprue through a prospective study involving 40 patients. They concluded that although early celiac sprue was challenging to diagnose, EC could accurately diagnose this disease. ${ }^{21}$ Following this study, Matysiak-Budnik et al. also researched this topic and reported typical EC findings of celiac sprue. ${ }^{22}$ Goda et al. reported an optimal dye for superficial non-ampullary duodenal epithelial tumors. They compared the image quality of methylene blue, toluidine blue, and crystal violet for normal duodenal mucosa and tumors. They concluded that $0.5 \%$ toluidine blue and $1 \%$ methylene blue were the optimal stains. ${ }^{23}$ Kumagai et al. investigated duodenal lesions in patients with familial polyposis syndrome. They established two facts. Approximately $90 \%$ of lesions that were not stained with methy-

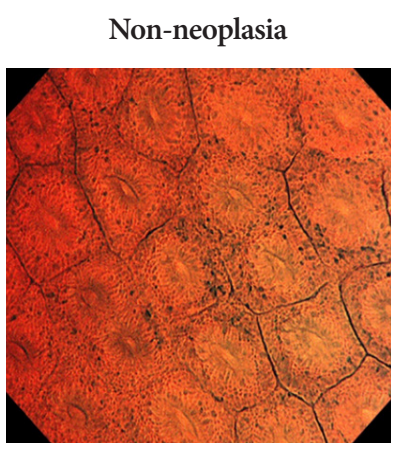

EC 1a

Roundish lumens

Uniform nuclei

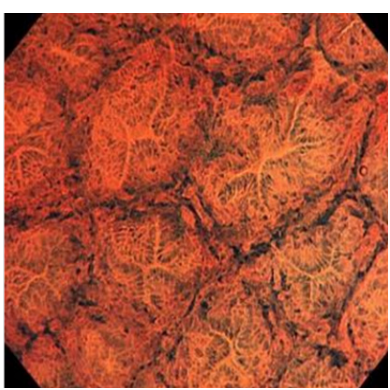

EC $1 b$

Serrated lumens Small granule nodules

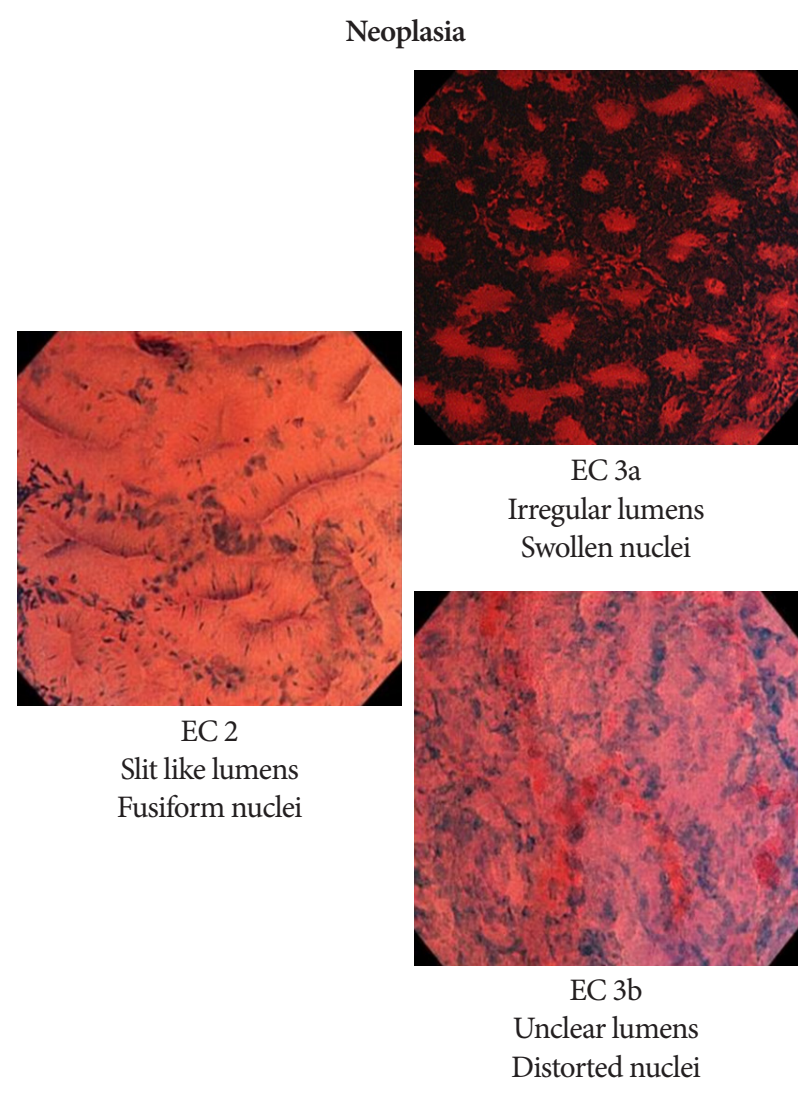

Fig. 2. Endocytoscopic classification for colorectal lesions, proposed by Kudo et al. ${ }^{5}$ Their classification is based on the structure of the crypts and the shape of the nuclei. EC, endocytoscopy. 
lene blue were pathologically diagnosed as neoplasms. Second, EC can identify cancerous lesions from many duodenal lesions of familial polyposis syndrome because cancerous lesions show enlarged oval-shaped nuclei. ${ }^{4}$

\section{EC FOR COLORECTAL NEOPLASMS}

EC for colorectal neoplasms is the most extensively investigated area. Several studies, including some prospective studies, have been reported. EC seems to have a highly accurate diagnostic performance that is comparable to the pathological diagnosis of colorectal neoplasms. However, almost all studies were conducted in Japan; therefore, it should be validated in another situation or another country.

Early on in this research field, some pilot studies investigated whether EC can visualize the cellular atypia of colorectal lesions as well as precedent esophageal disease. ${ }^{24,25}$ Subsequently, Kudo's group established an endoscopic image classification of colorectal lesions (EC classification, Fig. 2). The EC classification has three tiers, EC1 corresponds to non-neoplasia, EC2 corresponds to adenoma, and EC3 corresponds to cancerous tissue. Furthermore, EC1 and EC3 are subdivided into two classes: EC1a for normal mucosae, EC1b for hyperplastic polyps, EC3a for intramucosal or slightly invasive submucosal cancer, and EC3b for deep submucosal cancer., ${ }^{3,26}$ The diagnostic performance of the EC classification has been confirmed in some studies. There are two prospective trials regarding the differential diagnosis between neoplasms and non-neoplasms. Mori et al. conducted a randomized control trial to elucidate that EC is non-inferior to standard biopsy by comparing the pathological diagnosis. In total, 203 lesions were analyzed, and the diagnostic accuracy of EC for neoplasms was $94.1 \%$, which was non-inferior to standard biopsy (96.0\% accuracy) ${ }^{27} \mathrm{Ut}$ sumi et al. conducted a similar prospective trial and reported that EC had an accuracy of $96.8 \%$ for diagnosing adenoma. ${ }^{28}$ The paper is commendable in that it is the first prospective study reported by a research team other than developers. Kudo et al. also reported the efficacy for diagnosing sessile serrated lesion, ${ }^{29}$ identification of desmoplastic reaction, ${ }^{30}$ identification of tumor pathological grade, ${ }^{31}$ prediction of the invasive depth of colorectal cancer, ${ }^{32,33}$ and distinguishing low-grade from high-grade adenoma. ${ }^{34}$

In all the aforementioned studies, the diagnostic performance was based on methylene blue staining, which is sometimes time-consuming and cumbersome. In this regard, Kudo et al. reported a novel observation technique and classification using narrow-band imaging $(\mathrm{NBI})^{35,36}$ (Fig. 3). EC with NBI could visualize the microvessels on the surface of the lesions and provided accurate pathological prediction without the use of a dye solution. It seemed to be an optimal and first choice of diagnosis using EC, although the diagnostic performance of EC with NBI was relatively lower than that of methylene blue staining.

\section{EC FOR INFLAMMATORY BOWEL DISEASE (IBD)}

Several studies have reported the clinical efficacy of EC in patients with IBD. Clinicians must evaluate not only the histological inflammation grade but also colitis-associated neopla-

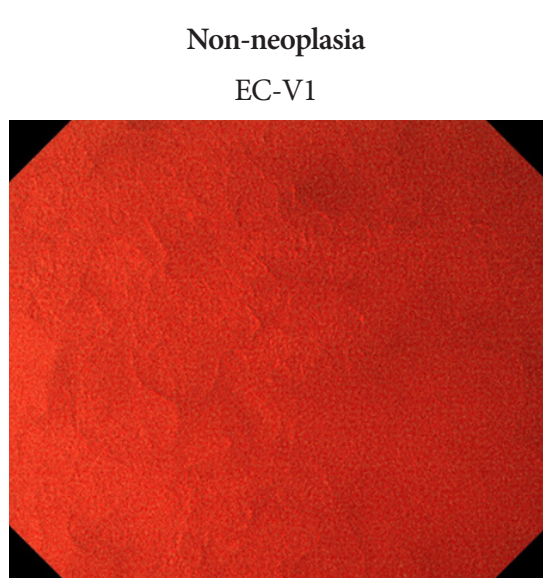

Obscure surface microvessels

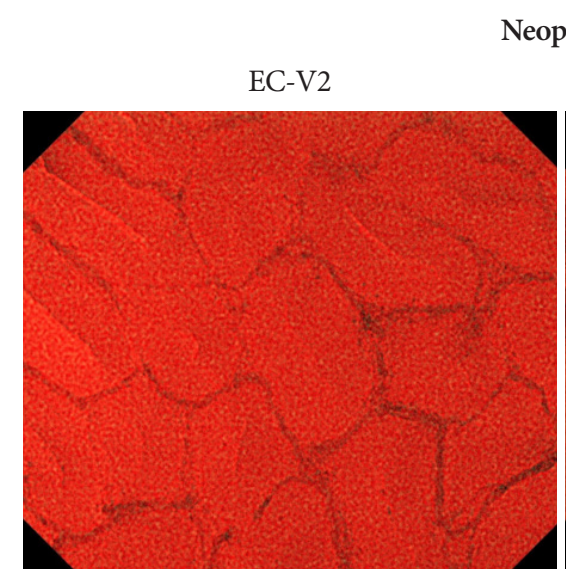

Clearly observed surface microvessels of a uniform caliber and arrangement
Neoplasia

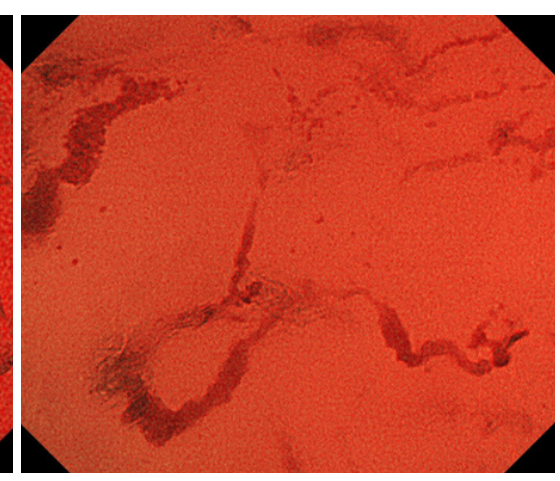

Dilated surface microvessels of a non-homogenous caliber

Fig. 3. Endocytoscopic classification based on narrow-band imaging, proposed by Kudo et al. ${ }^{34}$ This classification is based on the shape and arrangement of the surface microvessels. EC, endocytoscopy. 
sia during surveillance colonoscopy for patients with IBD. To evaluate these findings, biopsy and pathological diagnosis are currently mandatory. In addition to other fields, the role of EC in patients with IBD is the optical biopsy. Some studies have shown that EC can predict histopathological findings. Furthermore, the findings of EC seemed to correlate with clinical relapse in patients with IBD.

Bessho et al. first investigated the relationship between the EC findings and Matts' histological grading for patients with ulcerative colitis (UC) and established the EC system score (ECSS). The ECSS includes three factors: the shape of the crypts, distance between the crypts, and vessels. They conclud- ed that the ECSS showed a strong correlation with Matts' histological grade. ${ }^{37,38}$ Neumann et al. reported more detailed observations of infiltrated inflammatory cells. They prospectively observed the mucosa of 40 patients with IBD and concluded that EC could precisely identify the various types of infiltrated inflammatory cells (e.g., neutrophils, eosinophils, basophils). ${ }^{39}$ Maeda et al. approached this topic using NBI technology. They classified the microvessel findings of mucosae in patients with UC into three categories (i.e., vessels were 'obscure,' 'visible' or 'dilated'). These findings predicted acute inflammation with an accuracy of $92.3 \%$, which was superior to that predicted by conventional endoscopy. ${ }^{40}$

\section{Capillary index (0-3)}

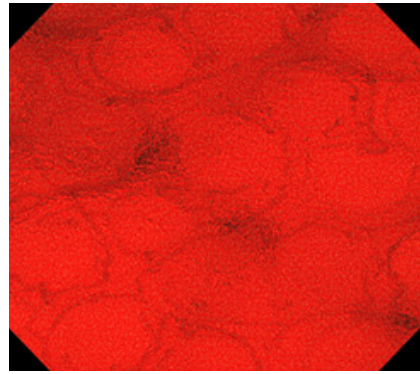

0: normal

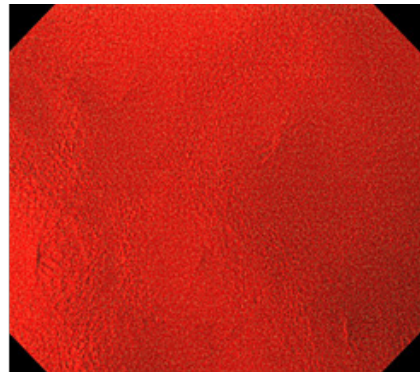

0: unrecognizable

Crypt index (0-2)

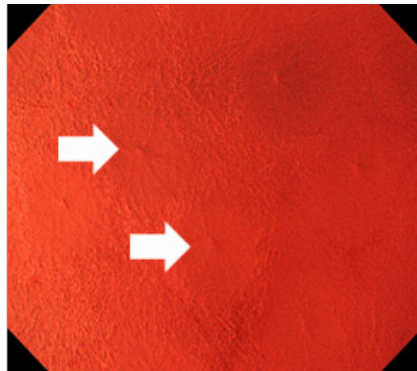

0: normal or unclear

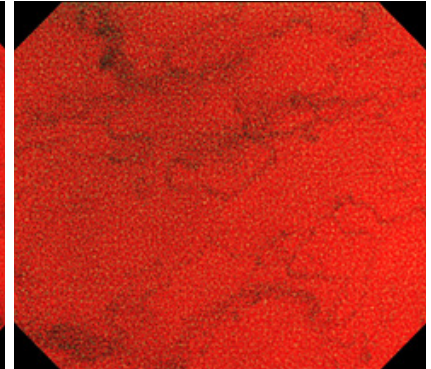

1: deformed

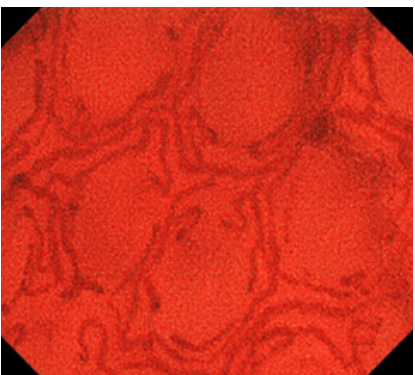

1: dilated

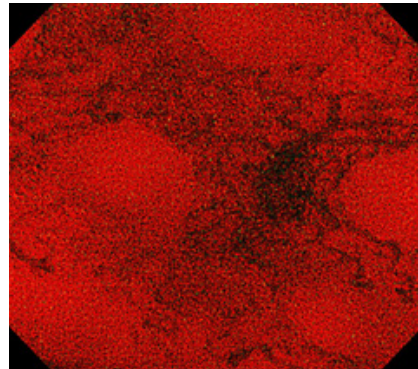

2: densely

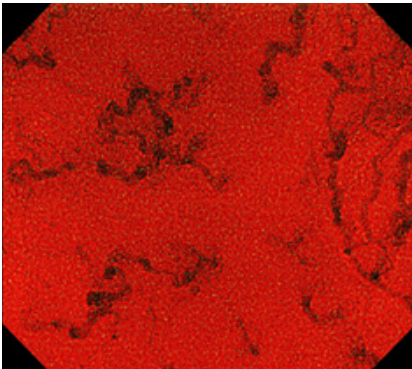

3: deformed+dilated

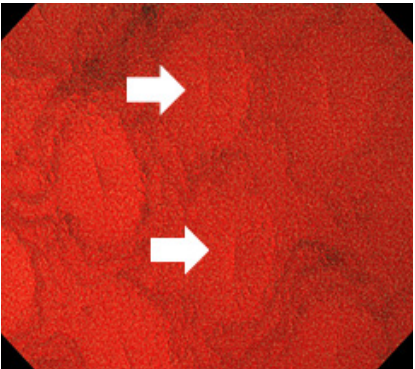

1: linear

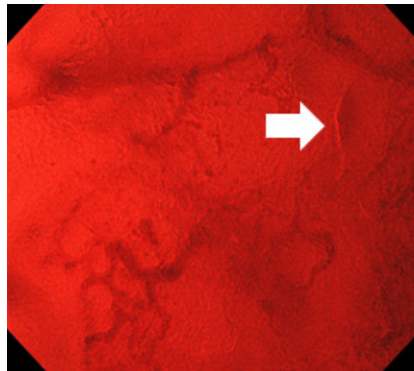

2: twist

ICC index (0-5): Capillary index (0-3)+Crypt index (0-2)

Fig. 4. The endocytoscopic scoring system for ulcerative colitis, proposed by Maeda et al. ${ }^{42}$ The intramucosal capillary/crypt (ICC) index comprises a capillary index and a crypt index. We defined the capillary index as follows: 0 , normal or unrecognizable; 1 , deformed or dilated; 2 , dense; 3 , deformed and dilated and the crypt index as follows: 0 , normal or unclear; 1 , linear; 2 , twisted. EC, endocytoscopy; ICC, intramucosal capillary/crypt. 
In addition to precise histological predictions, recent research has focused on whether endoscopic findings can predict the recurrence of UC. Precise prediction of disease relapse among patients with UC may assist in the decision on treatment strategy. Nishiyama et al. ${ }^{41}$ and Ueda et al. ${ }^{42}$ investigated this topic using EC. Although these two studies used different image classifications, patients who were judged to have no inflammatory activity or mild inflammation by EC were less likely to experience recurrence of UC. Maeda et al. reported the intramucosal capillary/crypt (ICC) index to evaluate the severity of inflammation in patients with UC (Fig. 4). Their ICC index is a scoring system based on the findings of EC with NBI. They concluded that the ICC index could stratify the risk of clinical relapse of $\mathrm{UC}^{43}$ Another interesting research topic is the identification of colitis-associated neoplasia using EC. Kudo et al. recently reported a pilot study that investigated the additional diagnostic effect of $\mathrm{EC}$ on conventional pit pattern diagnosis. In this study, pit pattern diagnosis plus EC provided significantly higher accuracy than conventional diagnosis. ${ }^{5}$ However, this was a retrospective single-center study; hence, we must wait for further studies regarding the diagnostic performance for UC-associated neoplasia.

\section{CONCLUSIONS}

We reviewed the clinical efficacy of EC for gastrointestinal diseases. By far, EC seems to provide accurate histopathological predictions as per the design concept. However, several limitations remain to be investigated. First, almost all studies were reported from a single Asian country; therefore, further validation is needed. Second, although maneuvering the magnifying endoscopy, including endocytoscope, requires training, no research is aimed at the learning curve to taking endocytoscopic images. Therefore, whether an endocytoscopic image can be constantly captured by non-experts should be investigated. Third, the cost-effectiveness of EC remains unclear. EC requires additional costs (equipment and dye) compared with conventional endoscopy. It is necessary to examine whether the improvement of diagnostic accuracy by EC (by only a few percent) contributes to cost-effectiveness. Fourth, skills regarding EC and image classification systems are still not standardized. If these limitations are solved, artificial intelligence ${ }^{44-47}$ might contribute to its dissemination and provide unified, highly accurate diagnoses even when used by non-experts.

In conclusion, EC opens a door to accurate optical biopsy; however, clinical evidence is still limited. Further validation studies are needed for the widespread use of this technology.

\section{Conflicts of Interest}

Shin-ei Kudo, Masashi Misawa, and Yuichi Mori received speaking honoraria from Olympus Corporation and have an ownership interest in the products of Cybernet Systems.

Funding

This research was supported by JSPS KAKENHI (grant number 19K17504).

Acknowlegments

We appreciate Shimamura’s research group for providing Figure 1.

Author Contributions

Conceptualization: Masashi Misawa, Shin-ei Kudo

Data curation: Yuki Takashina, Yoshika Akimoto

Funding acquisition: MM

Methodology: MM

Writing-original draft: MM

Writing-review\&editing: SK, Yasuharu Maeda, Yuichi Mori, Toyoki Kudo, Kunihiko Wakamura, Hideyuki Miyachi, Fumio Ishida, Haruhiro Inoue

ORCID

$\begin{array}{ll}\text { Masashi Misawa: } & \text { https://orcid.org/0000-0002-8520-2036 } \\ \text { Shin-ei Kudo: } & \text { https://orcid.org/0000-0002-4268-1217 } \\ \text { Yuki Takashina: } & \text { https://orcid.org/0000-0002-2473-7520 } \\ \text { Yoshika Akimoto: } & \text { https://orcid.org/0000-0002-4426-4628 } \\ \text { Yasuharu Maeda: } & \text { https://orcid.org/0000-0002-4820-5959 } \\ \text { Yuichi Mori: } & \text { https://orcid.org/0000-0003-2262-0334 } \\ \text { Toyoki Kudo: } & \text { https://orcid.org/0000-0002-2953-9603 } \\ \text { Kunihiko Wakamura: } & \text { https://orcid.org/0000-0003-0000-2092 } \\ \text { Hideyuki Miyachi: } & \text { https://orcid.org/0000-0002-8404-0899 } \\ \text { Fumio Ishida: } & \text { https://orcid.org/0000-0003-4962-4845 } \\ \text { Haruhiro Inoue: } & \text { https://orcid.org/0000-0002-0551-7274 }\end{array}$

Supplementary Materials

Video 1. Standard maneuver to obtain an ultra-magnified image. First, the endoscopist contacted the distal lens of the endocytoscope with the target lesion and then completely pulled down the magnification lever (https:// doi.org/10.5946/ce.2021.165.v001).

Video 2. Standard methylene blue staining technique for colorectal lesions. After conventional pit pattern diagnosis using crystal violet staining, the endoscopist dripped methylene blue through the catheter. After a minute, the endoscopist washed away the mucous and the remaining dye. It is important to wait for a minute to obtain a clear cellular image (https://doi. org/10.5946/ce.2021.165.v002).

\section{REFERENCES}

1. Inoue $\mathrm{H}$, Kazawa $\mathrm{T}$, Sato $\mathrm{Y}$, et al. In vivo observation of living cancer cells in the esophagus, stomach, and colon using catheter-type contact endoscope, "Endo-Cytoscopy system". Gastrointest Endosc Clin N Am 2004;14:589-594, x-xi.

2. Kumagai Y, Monma K, Kawada K. Magnifying chromoendoscopy of the esophagus: in-vivo pathological diagnosis using an endocytoscopy system. Endoscopy 2004;36:590-4.

3. Sasajima K, Kudo SE, Inoue H, et al. Real-time in vivo virtual histology of colorectal lesions when using the endocytoscopy system. Gastrointest Endosc 2006;63:1010-1017.

4. Kumagai Y, Yamamoto E, Higashi M, et al. Endocytoscopic observation 
with methylene blue staining for duodenal neoplasms associated with familial adenomatous polyposis. Sci Rep 2020;10:19221.

5. Kudo SE, Maeda Y, Ogata N, et al. Combined endocytoscopy with pit pattern diagnosis in ulcerative colitis-associated neoplasia: pilot study. Dig Endosc 2021 Feb 28 [Epub]. https://doi.org/10.1111/den.13964.

6. Ichimasa K, Kudo SE, Mori Y, et al. Double staining with crystal violet and methylene blue is appropriate for colonic endocytoscopy: an in vivo prospective pilot study. Dig Endosc 2014;26:403-408.

7. Minami H, Inoue H, Yokoyama A, et al. Recent advancement of observing living cells in the esophagus using CM double staining: endocytoscopic atypia classification. Dis Esophagus 2012;25:235-241.

8. Inoue $\mathrm{H}$, Sasajima K, Kaga M, et al. Endoscopic in vivo evaluation of tissue atypia in the esophagus using a newly designed integrated endocytoscope: a pilot trial. Endoscopy 2006;38:891-895.

9. Kumagai Y, Kawada K, Yamazaki S, et al. Endocytoscopic observation for esophageal squamous cell carcinoma: can biopsy histology be omitted? Dis Esophagus 2009;22:505-512.

10. Kumagai $Y$, Kawada K, Yamazaki S, et al. Current status and limitations of the newly developed endocytoscope GIF-Y0002 with reference to its diagnostic performance for common esophageal lesions. J Dig Dis 2012;13:393-400.

11. Kumagai Y, Kawada K, Higashi M, et al. Endocytoscopic observation of various esophageal lesions at $\times 600$ : can nuclear abnormality be recognized? Dis Esophagus 2015;28:269-275.

12. Shimamura Y, Inoue H, Rodriguez de Santiago E, et al. Diagnostic yield of fourth-generation endocytoscopy for esophageal squamous lesions using a modified endocytoscopic classification. Dig Endosc 2020 Dec 15 [Epub]. https://doi.org/10.1111/den.13914.

13. Eleftheriadis $\mathrm{N}$, Inoue $\mathrm{H}$, Ikeda $\mathrm{H}$, et al. Endocytoscopic visualization of squamous cell islands within Barrett's epithelium. World J Gastrointest Endosc 2013;5:174-179.

14. Tomizawa Y, Iyer PG, Wongkeesong LM, et al. Assessment of the diagnostic performance and interobserver variability of endocytoscopy in Barrett's esophagus: a pilot ex-vivo study. World J Gastroenterol 2013;19:8652-8658.

15. Eberl T, Jechart G, Probst A, et al. Can an endocytoscope system (ECS) predict histology in neoplastic lesions? Endoscopy 2007;39:497-501.

16. Isomoto $\mathrm{H}$, Matsushima $\mathrm{K}$, Hayashi $\mathrm{T}$, et al. Endocytoscopic findings of lymphomas of the stomach. BMC Gastroenterol 2013;13:174.

17. Chiu PWY, Ng EKW, To KF, et al. Recognition of goblet cells upon endocytoscopy indicates the presence of gastric intestinal metaplasia. Dig Endosc 2014;26:52-56.

18. Kaise M, Kimura R, Nomura K, et al. Accuracy and concordance of endocytoscopic atypia for the diagnosis of gastric cancer. Endoscopy 2014;46:827-832.

19. Kaise M, Ohkura Y, lizuka T, et al. Endocytoscopy is a promising modality with high diagnostic accuracy for gastric cancer. Endoscopy 2015;47:19-25.

20. Sato $H$, Inoue $H$, Hayee B, et al. In vivo histopathology using endocytoscopy for non-neoplastic changes in the gastric mucosa: a prospective pilot study (with video). Gastrointest Endosc 2015;81:875-881.

21. Pohl H, Rösch T, Tanczos BT, Rudolph B, Schlüns K, Baumgart DC. Endocytoscopy for the detection of microstructural features in adult patients with celiac sprue: a prospective, blinded endocytoscopy-conventional histology correlation study. Gastrointest Endosc 2009;70:933941.

22. Matysiak-Budnik T, Coron E, Mosnier J-F, Le Rhun M, Inoue H, Galmiche J-P. In vivo real-time imaging of human duodenal mucosal structures in celiac disease using endocytoscopy. Endoscopy 2010;42:191196.

23. Goda K, Dobashi A, Yoshimura N, et al. Dye solution optimizing staining conditions for in vivo endocytoscopy for normal villi and superficial epithelial tumors in the duodenum. Ann Gastroenterol 2019;32:378386.

24. Kodashima S, Fujishiro M, Takubo K, et al. Ex-vivo study of high-magnification chromoendoscopy in the gastrointestinal tract to deter- mine the optimal staining conditions for endocytoscopy. Endoscopy 2006;38:1115-1121.

25. Cipolletta L, Bianco MA, Rotondano G, et al. Endocytoscopy can identify dysplasia in aberrant crypt foci of the colorectum: a prospective in vivo study. Endoscopy 2009;41:129-132.

26. Kudo SE, Wakamura K, Ikehara N, Mori Y, Inoue H, Hamatani S. Diagnosis of colorectal lesions with a novel endocytoscopic classification - a pilot study. Endoscopy 2011;43:869-875.

27. Mori Y, Kudo SE, Ikehara N, et al. Comprehensive diagnostic ability of endocytoscopy compared with biopsy for colorectal neoplasms: a prospective randomized noninferiority trial. Endoscopy 2013;45:98-105.

28. Utsumi T, Sano Y, Iwatate M, et al. Prospective real-time evaluation of diagnostic performance using endocytoscopy in differentiating neoplasia from non-neoplasia for colorectal diminutive polyps $(\leq 5 \mathrm{~mm})$. World J Gastrointest Oncol 2018;10:96-102.

29. Kutsukawa M, Kudo SE, Ikehara N, et al. Efficiency of endocytoscopy in differentiating types of serrated polyps. Gastrointest Endosc 2014;79:648-656.

30. Sugihara $\mathrm{Y}$, Kudo SE, Miyachi $\mathrm{H}$, et al. In vivo detection of desmoplastic reaction using endocytoscopy: a new diagnostic marker of submucosal or more extensive invasion in colorectal carcinoma. Mol Clin Oncol 2017;6:291-295.

31. Sako T, Kudo SE, Miyachi H, et al. A novel ability of endocytoscopy to diagnose histological grade of differentiation in T1 colorectal carcinomas. Endoscopy 2018;50:69-74.

32. Kudo T, Kudo SE, Wakamura K, et al. Diagnostic performance of endocytoscopy for evaluating the invasion depth of different morphological types of colorectal tumors. Dig Endosc 2015;27:754-761.

33. Kudo T, Kudo SE, Mori Y, et al. Classification of nuclear morphology in endocytoscopy of colorectal neoplasms. Gastrointest Endosc 2017;85:628-638.

34. Kudo T, Suzuki K, Mori Y, et al. Endocytoscopy for the differential diagnosis of colorectal low-grade adenoma: a novel possibility for the "resect and discard" strategy. Gastrointest Endosc 2020;91:676-683.

35. Kudo SE, Misawa M, Wada Y, et al. Endocytoscopic microvasculature evaluation is a reliable new diagnostic method for colorectal lesions (with video). Gastrointest Endosc 2015;82:912-923.

36. Nakamura H, Kudo SE, Misawa M, et al. Evaluation of microvascular findings of deeply invasive colorectal cancer by endocytoscopy with narrow-band imaging. Endosc Int Open 2016;4:E1280-E1285.

37. Bessho R, Kanai T, Hosoe N, et al. Correlation between endocytoscopy and conventional histopathology in microstructural features of ulcerative colitis. J Gastroenterol 2011;46:1197-1202.

38. Nakazato Y, Naganuma M, Sugimoto S, et al. Endocytoscopy can be used to assess histological healing in ulcerative colitis. Endoscopy 2017;49:560-563.

39. Neumann H, Vieth M, Neurath MF, Atreya R. Endocytoscopy allows accurate in vivo differentiation of mucosal inflammatory cells in IBD: a pilot study. Inflamm Bowel Dis 2013;19:356-362.

40. Maeda Y, Ohtsuka K, Kudo SE, et al. Endocytoscopic narrow-band imaging efficiency for evaluation of inflammatory activity in ulcerative colitis. World J Gastroenterol 2015;21:2108-2115.

41. Nishiyama S, Oka S, Tanaka S, et al. Clinical usefulness of endocytoscopy in the remission stage of ulcerative colitis: a pilot study. J Gastroenterol 2015;50:1087-1093.

42. Ueda N, Isomoto H, Ikebuchi Y, et al. Endocytoscopic classification can be predictive for relapse in ulcerative colitis. Medicine (Baltimore) 2018;97:e0107.

43. Maeda Y, Kudo SE, Ogata N, et al. Endocytoscopic intramucosal capillary network changes and crypt architecture abnormalities can predict relapse in patients with an ulcerative colitis Mayo endoscopic score of 1. Dig Endosc 2020;32:1082-1091.

44. Kudo SE, Misawa M, Mori Y, et al. Artificial intelligence-assisted system improves endoscopic identification of colorectal neoplasms. Clin Gas- 
troenterol Hepatol 2020;18:1874-1881.e2.

45. Maeda Y, Kudo SE, Mori Y, et al. Fully automated diagnostic system with artificial intelligence using endocytoscopy to identify the presence of histologic inflammation associated with ulcerative colitis (with video). Gastrointest Endosc 2019;89:408-415.

46. Misawa M, Kudo SE, Mori Y, et al. Artificial intelligence-assisted pol- yp detection for colonoscopy: initial experience. Gastroenterology 2018;154:2027-2029.e3.

47. Mori Y, Kudo SE, Misawa M, et al. Real-time use of artificial intelligence in identification of diminutive polyps during colonoscopy: a prospective study. Ann Intern Med 2018;169:357-366. 